\title{
Alitretinoin - its use in intractable hand eczema and other potential indications
}

\section{Bibi Petersen Gregor BE Jemec}

Dept of dermatology, Health Sciences faculty, University of Copenhagen, Roskilde Hospital, Denmark
Correspondence: Bibi Petersen Dept of dermatology, Health Sciences faculty, University of Copenhagen, Roskilde Hospital, Denmark Fax +45 47322698 Email bibp@regionsjaelland.dk

\begin{abstract}
Chronic hand eczema is a significant cause of morbidity. A number of treatments are traditionally used, and often useful, but in spite of this a sizeable group of patients develop chronic recalcitrant hand eczema. Retinoids are known to influence keratinization and inflammation, and acitretin has shown some effect in the treatment of chronic hand eczema. Alitretinoin (9-cis-retinoic acid) is a panagonist retinoid binding to all six known retinoid receptors (RAR- $\alpha,-\beta,-\gamma$ and RXR- $\alpha,-\beta,-\gamma)$. Several studies have been carried out with this new drug, and it has been shown to be effective in $28 \%$ to $89 \%$ of patients with previously intractable hand eczema. In addition, alitretinoin appears to have some potential in the treatment of AIDS-related Kaposi sarcoma. Attempts to use the drug in secondary prophylaxis has shown some promise in former tobacco smokers, whereas no effect was seen in patients with cervical intraepithelial neoplasia. Currently, the primary therapeutic potential of alitretinoin appears to be the treatment of chronic recalcitrant hand eczema, which also forms a large and hitherto neglected group of patients.
\end{abstract}

Keywords: alitretinoin, hand, eczema, dermatitis

Chronic hand dermatitis (CHD) is invalidating socially and occupationally, with an increased risk of prolonged sick leave and unemployment. ${ }^{1}$

CHD is characterized by erythema, vesicles, papules, edema, scaling, fissures, hyperkeratosis, and symptoms of itch and pain, and is classified into the following subgroups based on etiology and morphology: irritant contact dermatitis, allergic contact dermatitis, atopic dermatitis, hyperkeratotic eczema, nummular hand eczema, endogen eczema and pompholyx (dyshidrotic eczema), with no existing agreement of classification. ${ }^{1}$

For patients with severe CHD the treatment options have hitherto been limited. Besides the usually advise of avoiding allergens and irritants, treatment with emollients, corticosteroids, UV-irradiation, ionizing radiation and immunosuppressants have been used with varying effect. A systemic immunosuppressive treatment is a possibility for shorter periods, but is not suitable for long-term treatment, and therefore an effective alternative is needed. Previous studies have suggested that retinoids may provide a useful non-immunosuppressive alternative treatment. Retinoids are known to have a normalizing effect on cell differentiation, to be antiproliferative and to regulate cell-apoptosis. The present retinoid that is indicated in the treatment of chronic hand eczema is acitretin, although the effect has been limited.

The new pan-retinoid-receptor agonist 9-cis-retinoid acid has been experimentally used in the treatment of different diseases. Alitretinoin is a panagonist retinoid, which is capable of binding to all six known retinoid receptors (RAR- $\alpha,-\beta,-\gamma$ and RXR- $\alpha,-\beta,-\gamma)$, in contrast to tretinoin that primarily binds to RAR- $\alpha$, acitretin that activates but does not bind to RARs and 13-cis-retinoid acid that shows low affinity for RARs. ${ }^{2}$ Therefore alitretinoin is expected to be effective in a different spectrum of diseases. Good results have also been shown with alitretinoin in the treatment for CHD, 
Kaposi's sarcoma (KS) and a moderate effect in the therapy of specific cancers has been demonstrated. ${ }^{3-15}$

The aim of this work was to review recent advances in the treatment of CHD synonymous with chronic hand eczema, and to review previous trials of the retinoid alitretinoin (9 cis-retinoic acid (RA)) in the treatment of this and other diseases.

\section{Chronic hand dermatitis and treatment}

In the therapy of CHD the first step is skin protection and prophylaxis; using gloves, avoiding irritants and allergens and using emollients. Topical application with corticosteroid creams and ointments is the mainstay in treatment of CHD; alternatively or in addition to this, topical immunomodulators, coal tar, ultraviolet (UV) light and ionizing radiation are used topically. Treatment of CHD with corticosteroids is well documented. ${ }^{16}$ Because many patients with CHD often do not respond adequately to topical treatment, it is frequently necessary to change to a systemic immunosuppressive treatment.

The effect of topical calcineurin inhibitors (TCIs) has been evaluated in two studies. An open prospective study with 29 patients showed over 50\% reduction in 59\% of patients after 4 weeks of treatment with tacrolimus ointment (Protopic $^{\circledR} 0.1 \%$ ) and 44\% were cleared of hand eczema at the final visit. ${ }^{17}$ Another small randomized study evaluated the effect of tacrolimus $0.1 \%$ versus mometasone furuate $0.1 \%$ in the treatment of dyshidrotic eczema on palms and soles. Tacrolimus was found equally effective to mometasone furuate in chronic dyshidrotic eczema of the hands, but no effect was seen in plantar eczema. ${ }^{18}$

The effect of UV-light therapy has been investigated, and both irradiation with UVA-1 and UVB have been found effective. ${ }^{19,20}$

Several studies have documented the effect of local PUVA treatment. ${ }^{21,22}$ Methotrexate has been described as an effective treatment and steroid-sparing agent in recalcitrant palmoplantar pompholyx. ${ }^{23}$

A good effect of cyclosporine has been shown in a study including patients with eczematous diseases including CHD and a possible long-term remission after withdrawal of cyclosporine was suggested. ${ }^{24}$ Another study supported these results. ${ }^{25}$

\section{Chronic hand dermatitis and retinoids}

Retinoids exert their effect by binding to specific nuclear receptors and thereby regulate cell growth and cellular proliferation, differentiation and apoptosis. This effect is a class effect, but each retinoid has a higher affinity for specific receptors. Retinoid receptors are divided into two groups: RAR- $\alpha, \beta, \gamma$ and RXR- $\alpha, \beta, \gamma$.

In the skin the predominant retinoid receptor is the RAR- $\gamma /$ RXR- $\alpha$ heterodimer. ${ }^{26}$

Topical treatment with bexarotene gel for 22 weeks has been investigated in an open label randomized (into 3 treatment arms) study including 55 patients with CHD. According to the physician assessment (PA) bexarotene gel (BG) was comparative in effect to bexarotene gel combined with topical mometasone furoate ointment $0.1 \%(B G+M F)$ and superior to $\mathrm{BG}$ in combination with topical hydrocortisone ointment $1 \%(\mathrm{BG}+\mathrm{HC})$. An improvement of over $50 \%$ was seen in $79 \%$ of the $\mathrm{BG}$ arm, $77 \%$ in the $\mathrm{BG}+\mathrm{MF}$ arm and $50 \%$ of the $\mathrm{BG}+\mathrm{HC}$ arm. These results were supported by the secondary end-points: The Eczema Area and Severity Index (EASI) and The Hand EASI (HEASI). ${ }^{27}$

In a single-blinded randomized study treatment of hyperkeratotic hand eczema with $30 \mathrm{mg}$ of acitretin daily or placebo, acitretin was found to be statistically significantly more effective than placebo. There was an overall $51 \%$ reduction in clinical symptoms after 4 weeks in the acitretin group compared with a $9 \%$ reduction in the placebo group. ${ }^{28}$

\section{Chronic hand dermatitis and alitretinoin}

Alitretinoin (9-cis RA) is known to be a panagonist retinoid with high affinity to all six retinoid receptors, in contrast to other known retinoids which target mainly RARreceptors. ${ }^{2,26}$ Therefore it is expected to exert its effect in a different way than previously used retinoids such as acitretin.

In 1999 alitretinoin was described as an effective treatment for CHD after an open-label study with oral 9-cis-retinoic acid. It included 38 patients with the following diagnoses: pompholyx, hyperkeratotic eczema, fingertip eczema and discoid eczema, all with unsatisfactory response to previous treatments. All patients were given a once daily oral dose of 20 or $40 \mathrm{mg}$ alitretinoin, in a mean duration of 2.3 months (range $1-5$ months). Therapeutic response (TR) was conducted with a 4 -point scale $(0=$ none, $1=$ slight, $2=$ moderate, $3=$ marked, $4=$ severe) recorded for each of the lesions and symptoms: erythema, papules, desquamation, hyperkeratosis, rhagades and pruritus; thereafter a reduction was evaluated in percent. A reduction in total lesion symptom score of $0 \%$ to $20 \%=$ no therapeutic response, $21 \%$ to $40 \%=$ slight $\mathrm{TR}, 41 \%$ to $60 \%=$ moderate $\mathrm{TR}, 61 \%$ 
to $80 \%=\operatorname{good} \mathrm{TR}, 81 \%$ to $100 \%=$ very good $\mathrm{TR}$. In this study $89 \%$ showed a very good or good TR, 5.5\% a moderate TR and 5.5\% had no response. Headache, cheilitis, flushing and conjunctivitis were reported. Two patients had to be reduced from $40 \mathrm{mg}$ to $20 \mathrm{mg}$ because of flushing and one because of conjunctivitis; after dose reduction their side effects disappeared. Unsatisfactory effect was seen in 3 patients who received $20 \mathrm{mg}$ daily, but after increasing dose to $40 \mathrm{mg}$ daily, they all achieved a good response. The study suggested clinical effect on CHD and a manageable, dose-dependent profile of common side effects. ${ }^{3}$

A randomized, double blind, placebo-controlled, multicenter trial was conducted in 2001/2002 in 43 clinics in Europe, enrolling 319 patients with CHD (hyperkeratotic eczema, fingertip eczema, dyshidrotic eczema). Patients were randomized into 4 blocks receiving treatment with placebo or with alitretinoin $10 \mathrm{mg}, 20 \mathrm{mg}$ or $40 \mathrm{mg}$ once daily for 12 weeks. Twenty-four percent withdrew before completion, mainly caused by insufficient therapeutic response. The physician's global assessment (PGA) was primarily used to measure the results of the treatment, and secondary efficacy measures were the total lesion symptom score (TLSS) and patient's global assessment (PaGA). PGA was classified as severe/moderate/mild/almost clear or clear depending on the presence of erythema, scaling, hyperkeratosis/lichenification, vesiculation, edema, fissures and pruritus/pain; the intensity of the symptom; and the proportion of the hand surface involved. The assessment 'clear' was made in absence of all features, while the assessment of 'severe' required the following criteria to be met: at least one of the following to be moderate/severe: erythema, scaling, hyperkeratosis/lichenification; at least one severe of the following: vesiculation, edema, fissures, pain/pruritus; and more than $30 \%$ of the hand surface affected (see Ruzicka et $\mathrm{al}^{4,5}$ for additional detail). Responders according to PGA were $27 \%, 39 \%$, and $41 \%$, and $53 \%$ to placebo, at 10, 20 and $40 \mathrm{mg}$ day, respectively. Patients were regarded as responders if they were rated as clear or almost clear at week 12. The closed Cochran-Armitage trend test used on the PGA results revealed a significant and dosedependent effect $(\mathrm{p}<0.001 \%$ ) The TLSS also showed a dose dependent reduction in symptoms with a $-25,-59,-52$ and $-70,5$ reduction, respectively ( $\mathrm{p}<0.001$, JonckheereTerpstra trend test). Responders according to the PaGA were similar $(12 \%, 29 \%, 34 \%$ and $43 \%)$ and according to the chi-squared test significant at $\mathrm{p}=0.01$ for $10 \mathrm{mg} / \mathrm{day}$, $\mathrm{p}=0.002$ for $20 \mathrm{mg} /$ day and $\mathrm{p}<0.001$ for $40 \mathrm{mg} /$ day. During a 12 -week follow-up among the responders $26 \%$ relapsed and required prescription therapy. Generally alitretinoin was well tolerated, but $53 \%$ of the $40 \mathrm{mg} / \mathrm{d}$ group experienced adverse events, most frequently headache, dry lips/mouth, flushing and erythema. ${ }^{4}$

Another multicenter trial was conducted in Europe and Canada with enrolment of 1032 patients from 2004 to 2006. Patients were randomized in the ratio 1:2:2 to placebo, $10 \mathrm{mg}$ or $30 \mathrm{mg}$ of oral alitretinoin once daily for up to 24 weeks. Dose reduction was not allowed. The primary efficacy parameter was PGA which was performed at screening and every 4 weeks during treatment. Response was defined as 'clear' or 'almost clear'. Response rates were clearly dose dependent with 195/409 (48\%) responders in the $30 \mathrm{mg}$ alitretinoin group, $115 / 418(28 \%)$ in the $10 \mathrm{mg}$ group and $34 / 205$ (17\%) in the placebo group. Results of secondary efficacy parameters were consistent with the primary. The time to response was found significantly shorter in the $30 \mathrm{mg}$ group compared to the $10 \mathrm{mg}$ group. PaGA and median \% reduction in mTLSS and median \% reduction in extent of disease were all found significantly more reduced in the $30 \mathrm{mg}$ group than in the placebo group. The most frequently adverse event was headache, leading to withdrawal of $4 \%$ of the patients in the $30 \mathrm{mg}$ group and $1 \%$ of the patients in both the $10 \mathrm{mg}$ group and the placebo group. The adverse events also comprised mucocutaneous events, hyperlipidemia and decreased thyroid stimulating hormone, all of these well known as retinoid class effects and, like headache, appeared to be dose dependent. ${ }^{5}$

The results of the studies are summarized in Table 1.

\section{Use of alitretinoin in pre-malignancy and malignancy}

Because retinoids have a normalizing effect on cell differentiation, are anti-proliferative and regulate cell-apoptosis, they have shown some promise in the treatment of cancer. ${ }^{29-32}$

\section{Treatment of former smokers}

Lung cancer is one of the leading causes of cancer death, with tobacco smoking as a major risk factor. Lung cancer risk remains elevated after smoking cessation, and ways to stop cell differentiation and prevent carcinogenesis in former smokers have been investigated.

In former smokers the loss of retinoic acid receptor beta (RAR- $\beta$ ) expression in the bronchial epithelium is considered a biomarker of incipient neoplasia. To investigate the potential effect of retinoids in re-establishing the RAR- $\beta$ expression, a randomized placebo controlled trial with 226 former smokers was conducted. Patients received either 9-cis-retinoic acid 
Table I Summarized results of clinical studies of alitretinoin in chronic hand dermatitis indicating a response rate of $27 \%$ to $89 \%$ depending on dosage and duration of treatment

\begin{tabular}{|c|c|c|c|c|}
\hline & Patients & Doses & Duration & Responders \\
\hline \multirow[t]{2}{*}{ Bollag and Ott ${ }^{3}$} & 38 Open-label & $20-40 \mathrm{mg}$ & 2.3 months (mean) & \\
\hline & & & & 89\% (total) TLSS \\
\hline \multirow[t]{2}{*}{ Ruzicka et $\mathrm{al}^{4}$} & 319 Randomized & $10-40 \mathrm{mg}$ & 12 weeks & \\
\hline & & & & $39 \%-41 \%-53 \%(10-20-40 \mathrm{mg})$ PGA \\
\hline Ruzicka et $\mathrm{al}^{5}$ & I032 Randomized & $10-30 \mathrm{mg}$ & -24 weeks & $28 \%-48 \%(10-30 \mathrm{mg})$ PGA \\
\hline
\end{tabular}

Abbreviations: PGA, physician's global assessment; TLSS, total lesion symptom score.

(RA) (100 mg), 13-cis-RA (1 mg/kg) plus $\alpha$-tocopherol or placebo. The effect was evaluated trough bronchoscopy and biopsies from 6 different locations before and after treatment, and the specimens were evaluated for squamous metaplasia, dysplasia and RAR- $\beta$-expression. A total of 177 subjects completed at least 3 months of therapy and the examination 3 months after; in these RAR- $\beta$-expression at baseline was found in $62.6 \%$. A significant increase in RAR- $\beta$ was found in the 9-cis-RA group, in which 15 subjects changed from negative to positive expression and 5 from positive to negative expression. These changes were not found to be significant in the other groups.

Metaplasia was found in $6.9 \%$ of all baseline samples and $29.6 \%$ of subjects. Dysplasia was found in 5 subjects. A significant percentage decline in subjects with metaplasia was found in the 9-cis-RA group, but not in the others, and not when quantified as a continuous variable (metaplasia index), where the total numbers of samples with metaplasia were evaluated. It was hypothesized that the restoration of RAR- $\beta$ reflects suppression of bronchial neoplasia. ${ }^{33}$

As described above the primary end-point was RAR$\beta$-expression, but later the group published an analysis of the anti-proliferative effect of the two retinoids trough expression of Ki-67. In the per-subject analyses treatment with 13-cis-RA plus $\alpha$-tocopherol was statistically significantly associated with a reduced cell-proliferation after 3 months, but treatment with 9-cis-RA was not. In the per-site analyses both groups showed a statistically significant decreased proliferation assessed by the Ki-67 labeling index. ${ }^{34}$

Because low serum concentrations of antioxidants is suspected to be associated with an increased risk of cancer, the same group conducted a retrospective analysis of the blood specimens taken before and after the treatment from the same randomized study, to determine whether 9-cis-RA could elevate the s- $\alpha$-tocopherol in former smokers. The arm receiving 13 -cis-RA plus $\alpha$-tocopherol was excluded from this analysis. The baseline s- $\alpha$-tocopherol in the 9-cis-RA group and the placebo group did not differ, but it was significantly associated with serum triglyceride level and age.

Serum $\alpha$-tocopherol significantly increased over time in the 9-cis-RA group, but not in the placebo group. ${ }^{35}$

\section{Kaposi's sarcoma}

Several studies have shown positive results with the treatment of KS with topical alitretinoin, as well as alitretinoin given orally. KS is a tumor caused by the human herpes virus 8 (HHV 8) and it is often associated with acquired immune deficiency syndrome (AIDS), but after the development of highly active antiretroviral therapy (HAART) the frequency of KS has declined.

In 1996-1997 the first randomized, double-blind, controlled, multicenter study of the safety and efficacy of topical alitretinoin was conducted in the US, enrolling 268 patients with AIDS and KS, with 134 patients receiving treatment with a topical alitretinoin gel $0.1 \%$ and 134 patients a vehicle gel. Patients had at least 6 lesions and applied the gel 3 times daily for the first 2 weeks and 4 times daily for the last 10 weeks of the study period.

During the study the lesions were assessed for area, height, color and pain with responses evaluated and defined by ACTG-criteria (Aids Clinical Trial Group). In total 34\% of patients receiving alitretinoin $0.1 \%$ gel achieved partial response (PR) and $0.7 \%$ complete response (CR), compared with $18 \%$ and $0 \%$, respectively, in the group receiving vehicle gel. After the blinded part of the trial, patients were offered to continue treatment in an open label study, and 14 weeks of treatment with alitretinoin gel gave a $50 \%$ response rate and 27 weeks a $75 \%$ response rate. ${ }^{6}$

A further phase 1 and 2 dose escalation study enrolled 115 AIDS patients with KS from 9 centers in an open-label study with alitretinoin gel. All patients received alitretinoin gel either $0.05 \%$ or $0.1 \%$ twice daily for the first 2 weeks, following an escalation dose every second week, until the most intensive, tolerated dose was reached. In total 
758 lesions were treated. The median time for treatment was 14 weeks. In the group of treated lesions $27 \%$ of patients had response to treatment, compared to control lesions with response in $11 \%$, according to the ACTG criteria. $^{7}$

Two non-randomized studies evaluated the effect of systemic alitretinoin in the treatment of AIDS-related KS. These phase II trials treated 60 and 57 patients with up to $140 \mathrm{mg} / \mathrm{m}^{2}$ escalating from $60 \mathrm{mg} / \mathrm{m}^{2}$, respectively. The median duration of therapy was 15 weeks in both trials, and the overall response rate was $37 \%$ and $39 \%$, using the ACTG criteria. Twenty-eight and 21 (37\%) patients withdrew from the study treatment because of adverse events (headache, skin toxicity, depression). Seven serious adverse events related to alitretinoin treatment were reported in 5 patients from one of the trials: pancreatitis, hypercalcinuria and mild renal insufficiency, anemia and severe headache. Cholesterol and triglyceride elevation were seen, but it is important to remember that protease inhibitors can have the same effect. Skin toxicity was cumulative, and levels of plasma cholesterol increased over time., ${ }^{8,9}$

A further randomized controlled trail supported the superiority of alitretinoin over vehicle in the treatment of KS. The group included 134 patients receiving either alitretinoin gel $0.1 \%$ or a vehicle gel twice daily for 12 weeks. In the alitretinoin group, response was favorable with $37 \%$ responders versus only $7 \%$ responders in the vehicle gel group. ${ }^{10}$

\section{Other malignancies}

Hematological malignancies have previously been treated with retinoids. After 9-cis-RA has been introduced, its effect in hematology is under investigation.

A group of 29 patients with non-hodgkin and refractory T-cell lymphomas were treated with 9-cis-RA as a single daily oral dose of $100 \mathrm{mg} / \mathrm{m}^{2}$, escalating to $140 \mathrm{mg} / \mathrm{m}^{2}$ after 2 weeks. One patient responded to treatment with complete remission, 3 patients had a partial response, 1 patient had a minor response, 9 patients were stable in disease, and 1 did not respond, but had progression in disease. Toxic effects resulted in discontinuation of treatment in 5 patients (headache, fatigue, and hypercalcemia) and required dose reduction in 8 patients. One patient died before he started treatment. Toxic effects reported were: dry skin, headache, xerostoma, fatigue, nausea, peripheral edema, fever of unknown origin, infection, dry eyes and cheilitis. ${ }^{11}$

In the US 18 patients diagnosed with acute promyelocytic leukemia (APL) were treated with 9-cis-RA in the period 1994-1996 in a non-randomized study. The patients received 30 to $230 \mathrm{mg} / \mathrm{m}^{2}$ as a single oral dose in the trial. Five were newly diagnosed and 4 of these achieved complete remission for 17 to 27 months. Thirteen patients were previously treated and 4 of these achieved complete remission in 14 to 40 months.

Headache and dry skin were the most common adverse reactions reported by $76 \%$ and $59 \%$, respectively. ${ }^{12}$

A multicenter phase II study included 30 patients with myelodysplatic syndromes, with 25 available for assessment. They received $60 \mathrm{mg} / \mathrm{m}^{2}$ daily in the first week, escalating to $100 \mathrm{mg} / \mathrm{m}^{2}$ in the second week and $140 \mathrm{mg} / \mathrm{m}^{2}$ in the third week.

One patient achieved complete remission and 4 patients responded with reduced transfusion requirements, making the overall response rate $20 \%$. Headache, dry skin, arthralgias and rashes were frequently reported adverse events in 77\%, 57\%, $30 \%$ and $23 \%$ of the patients, respectively. Hyperlipidemi and hypercalcemia were affected, supporting other observations of alitretinoin and retinoids in general. ${ }^{13}$

A group of 112 patients with cervical intraepithelial neoplasia $2 / 3$ (CIN 2/3) received a daily dose of 25 or $50 \mathrm{mg}$ of alitretinoin or placebo. There were no significant differences in regression among the 3 groups. Headache, fatigue and nausea were the most frequent reported adverse events. ${ }^{14}$

Another 41 patients with advanced cancer and previous failure to standard therapy were included in a safety study of alitretinoin and additionally the effect was evaluated, but no tumor responses were observed. ${ }^{15}$

\section{Discussion}

Hand eczema is a significant health problem which occurs both in association with underlying skin disease, eg, atopic eczema, as well as independently. ${ }^{36}$ There is a great need for new treatment options for intractable hand eczema, because it is a frequent and invalidating disease in the population. Recent advances in topical treatment have suggested that TCIs may be effective in mild-to-moderate CHD. ${ }^{17,18}$ The disadvantage of TCIs is their incompatibility with UV light irradiation and therapy, because of the potentially increased risk of non-melanoma skin cancer associated with this treatment. Topical retinoids have, however, also shown promise when good results appeared in a study of topically treatment of CHD with bexarotene gel. ${ }^{27}$

When topical treatments fail to control CHD, systemic immunosuppression is a possibility. Because of the side effects a non-immunosuppressive effective therapy is preferable, and acitretin has hitherto been the favored option for this. Acitretin is a retinoid that targets and activates RAR-receptors. In contrast, alitretinoin is a pan-retinoid-receptor agonist and therefore has a different target profile. Trials with alitretinoin 
(9-cis-RA) in the treatment of CHD have given promising results. ${ }^{3-5}$ Patients were treated with doses from 10 to $40 \mathrm{mg}$ daily and response was assessed according to PGA. When alitretinoin dose was taking into account, $28 \%$ to $53 \%$ of patients responded, with clearl dose dependency. In one study a durable remission was induced with a median of 5.5 to 6.2 months before responding patients relapsed with $75 \%$ of their initial symptoms, another found $26 \%$ of responders having a relapse during a 12-week follow up.,5

Because a retinoid has a normalizing effect on cell differentiation, proliferation and apoptosis, it has also been proposed as a treatment in neoplastic diseases. Several studies have been carried out with alitretinoin topically and systemically in the treatment of AIDS related KS. Respone rates from $27 \%$ to $39 \%$ were reported for a treatment period up to 15 weeks. ${ }^{6-10}$ As a systemic treatment, the adverse events limits its use in these patients, and in some patients the widespread affection of the skin, makes the topically treatment impractical. As a therapy in other malignant diseases the good results have been few. ${ }^{11-15}$

The most frequently reported adverse events were headache, dry lips/mouth, flushing, erythema, hyperlipidemia and decreased thyroid-stimulating hormone and seemed to be dose dependent. Headache remained the primary doselimiting adverse event, when alitretinoin was given at much higher doses as anti-cancer therapy.

In conclusion the results of published studies on alitretinoin suggests that the drug has a considerable therapeutic potential, with a good safety profile, for patients with severe hand eczema not responding to conventional topical therapy, whereas it has not been equally convincing as a potential anti-neoplastic agent, except for the treatment of Kaposi's sarcoma.

Additional relevant studies however need to be done to establish the full potential of this drug. Notably the potential for tachyphylaxis remains to be explored, as does the direct clinical comparison with known retinoids such as acitretin, which have also shown some potential in the treatment of CHD and skin cancer.

\section{Disclosures}

The authors have no conflicts of interest to disclose.

\section{References}

1. Diepgen TL, Agner T, Aberer W, et al. Management of chronic hand eczema. Contact Dermatitis. 2007;57(4):203-210.

2. Zouboulis CC. Retinoids - Which dermatological indications will benefit in the near future. Skin Pharmacol Appl Skin Physiol. 2001;14(5):303-315.
3. Bollag W, Ott F. Successful treatment of chronic hand eczema with oral 9-cis-retinoic acid. Dermatology. 1999;199(4):308-312.

4. Ruzicka T, Larsen FG, Galewicz D, et al. Oral alitretinoin (9-cis-retinoic acid) therapy for chronic hand dermatitis in patients refractory to standard therapy. Arch Dermatol. 2004;140(12):1453-1459.

5. Ruzicka T, Lynde CW, Jemec GBE, et al. Efficacy and safety of oral alitretinoin (9-cis retinoic acid) in patients with severe chronic hand eczema refractory to topical corticosteroids: results of a randomized, double-blind, placebo-controlled, multicentre trial. Br J Dermatol. 2008;158(4):808-817.

6. Walmsley S, Northfelt DW, Melosky B, et al. Treatment of AIDS-related cutaneous kaposis sarcoma with topical alitretinoin (9-cis-retinoic acid). J Acquir Immune Defic Syndr. 1999;22(3):235-246.

7. Duvic M, Friedman-Kien AE, Looney DJ, et al. Topical treatment of cutaneous lesions of acquired immunodeficiency syndrome-related kaposi sarcoma using alitretinoin gel. Results of phase 1 and 2 trials. Arch Dermatol. 2000;136(12):1461-1469.

8. Miles SA, Dezube BJ, Lee JY, et al. Antitumor activity of oral 9-cis-retinoic acid in HIV-associated kaposi's sarcoma. AIDS. 2002;16(3):421-429.

9. Aboulafia DM, Norris D, Henry D, et al. 9-cis-retinoic acid capsules in the treatment of AIDS-related kaposi sarcoma. Results of a phase 2 multicenter clinical trial. Arch Dermatol. 2003;139(2):178-186.

10. Bodsworth NJ. Bloch M, Bower M, Donnell D, Yocum R; for The International Panretin ${ }^{\circledR}$ Gel KS study group. Phase III vehicle-controlled, multi-centered study of topical alitretinoin gel $0.1 \%$ in cutaneous AIDS-related kaposi's sarcoma. Am J Clin Dermatol. 2001;2(2):77-87.

11. Younes A, Cristofanilli M, Mclaughlin P, et al. Experience with 9-cis-retinoic acid in patients with relapsed and refractory non-hodgkin's lymphoma. Leuk Lymphoma. 2000;40(1-2):79-85.

12. Soignet SL, Benedetti F, Fleischauer A, et al. Clinical study of 9-cis-retinoic acid (LGD1057) in acute promyelocytic leukemia. Leukemia. 1998;12(10):1518-1521.

13. Hofmann WK, Kell WJ, Fenaux P, et al. Oral 9-cis-retinoic acid (alitretinoin) in the treatment of myelodysplastic syndromes: results from a pilot study. Leukemia. 2000;14(9):1583-1588.

14. Alvarez RD, Conner MG, Weiss H, et al. The efficacy of 9-cis-retinoic acid (alitretinoin) as a chemopreventive agent for cervical dysplasia: results of a randomized double-blind clinical trial. Cancer Epidemiol Biomarkers Prev. 2003;12(2):114-119.

15. Rizvi NA, Marshall JL, Ness E, et al. Phase I study of 9-cis-retinoic acid (ALRT1057) in adults with advanced cancer. Clin Cancer Res. 1998;4(6):1437-1442.

16. Veien NK, Larsen PØ, Thestrup-Pedersen K, Schou G. Long-term, intermittent treatment of chronic hand eczema with mometasone furoate. Br J Dermatol. 1999;140(5):882-886.

17. Schliemann S, Kelterer D, Bauer A, et al. Tacrolimus ointment in the treatment of occupationally induced chronic hand dermatitis. Contact Dermatitis. 2008;58(5):299-306.

18. Schnopp C, Remling R, Möhrenschlager M, Weigl L, Ring J, Abeck D. Topical tacrolimus (FK506) and mometasone furoate in the treatment of dyshidrotic palmar eczema: A randomized, observer-blinded trial. J Am Acad Dermatol. 2002;46(1):73-77.

19. Sjövall P, Christensen OB. Local and systemic effect of UVB irradiation in patients with chronic hand eczema. Acta Derm Venereol. 1987;67(6):538-541.

20. Polderman MCA, Govaert JCM, Cessie SL, Pavel S. A double-blind placebo-controlled trial of UVA-1 in the treatment of dyshidrotic eczema. Clin Exp Dermatol. 2003;28(6):584-587.

21. Gritiyarangsan P, Sukhum A, Tresukosol P, Kullavanijaya P. Topical PUVA therapy for chronic hand eczema. J Dermatol. 1998;25(5):299-301.

22. Schempp CM, Müller H, Czech W, Schöpf E, Simon JC. Treatment of chronic palmoplantar eczema with bath-PUVA therapy. $J$ Am Acad Dermatol. 1997;36(5 pt 1):733-737. 
23. Egan CA, Rallis TM, Meadows KP, Krueger GG. Low-dose oral methotrexate treatment for recalcitrant palmoplantar pompholyx. $\mathrm{J} \mathrm{Am}$ Acad Dermatol. 1999;40(4):612-614.

24. Granlund H, Erkko P, Reitamo S. Long-term follow-up of eczema patients treated with cyclosporine. Acta Derm Venereol. 1998;78(1):40-43.

25. Reitamo S, Granlund H. Cyclosporin A in the treatment of chronic dermatitis of the hands. Br J Dermatol. 1994;130(1):75-78.

26. Cheng C, Michaels J, Scheinfeld N. Alitretinoin: a comprehensive review. Expert Opin Investig Drugs. 2008;17(3):437-443.

27. Hanifin JM, Stevens V, Sheth P, Breneman D. Novel treatment of chronic severe hand dermatitis with bexarotene gel. Br J Dermatol. 2004;150(3):545-553.

28. Thestrup-Pedersen K, Andersen KE, Menné T, Veien NK. Acta Derm Venereol. 2001;81(5)353-355.

29. Anolik JH, Giovanna JJD, Gaspari AA. Effect of isotetinoin therapy on natural killer cell activity in patients with xeroderma pigmentosum. Br J Dermatol. 1998;138(2):236-241.

30. Carneiro RV, Sotto MN, Azavedo LS, Ianhez LE, Rivitti EA. Acitretin and skin cancer in kidney transplanted patients. Clinical and histological evaluation and immunohistochemical analysis of lymphocytes, natural killer cells and langerhans' cells in sun exposed and sun protected skin. Clin Transplant. 2005;19(1):115-121.
31. Chen K, Craig JC, Shumack S. Oral retinoids for the prevention of skin cancers in solid organ transplant recipients: a systematic review of randomized controlled trials. Br J Dermatol. 2005;152(3):518-523.

32. Degos L. Differentiating agents in the treatment of leukemia. Leuk Res. 1990;14(8):717-719.

33. Kurie JM, Lotan R, Lee JJ, et al. Treatment of former smokers with 9 -cis-retinoic acid reverses loss of retinoic acid receptor- $\beta$ expression in the bronchial epithelium: results from a randomized placebo-controlled trial. J Natl Cancer Inst. 2003;95(3):206-214.

34. Hittelman WN, Liu DD, Kurie JM, et al. Proliferative changes in the brochial epithelium of former smokers treated with retinoids. $J$ Natl Cancer Inst. 2007;99(21):1603-1612.

35. Han J-Y, Liu DD, Lee JJ, et al. 9-cis-retinoic acid treatment increases serum concentrations of $\alpha$-tocopherol in former smokers. Clin Cancer Res. 2005;11(6):2305-2311.

36. Holm EA, Esmann S, Jemec GBE. The handicap of atopic dermatitis. $J$ Eur Acad Dermatol Venerol. 2006;20:255-260. 
